\title{
The rhodanese RhdA helps Azotobacter vinelandii in maintaining cellular redox balance
}

\author{
William Remelli', Angelo Cereda ${ }^{1}$, Jutta \\ Papenbrock $^{2}$, Fabio Forlani ${ }^{1}$ and Silvia Pagani, ${ }^{1, *}$ \\ ${ }^{1}$ Dipartimento di Scienze Molecolari Agroalimentari, \\ Università degli Studi di Milano, I-20133 Milano, Italy \\ ${ }^{2}$ Institute for Botany, Leibniz University Hannover, \\ Herrenhäuserstr. 2, D-30419 Hannover, Germany \\ * Corresponding author \\ e-mail: silvia.pagani@unimi.it
}

\begin{abstract}
The tandem domain rhodanese-homology protein RhdA of Azotobacter vinelandii shows an active-site loop structure that confers structural peculiarity in the environment of its catalytic cysteine residue. The in vivo effects of the lack of RhdA were investigated using an $A$. vinelandii mutant strain (MV474) in which the rhdA gene was disrupted by deletion. Here, by combining analytical measurements and transcript profiles, we show that deletion of the $r h d A$ gene generates an oxidative stress condition to which $A$. vinelandii responds by activating defensive mechanisms. In conditions of growth in the presence of the superoxide generator phenazine methosulfate, a stressor-dependent induction of rhdA gene expression was observed, thus highlighting that RhdA is important for $A$. vinelandii to sustain oxidative stress. The potential of RhdA to buffer general levels of oxidants in A. vinelandii cells via redox reactions involving its cysteine thiol is discussed.
\end{abstract}

Keywords: oxidative stress; transcriptomic analyses.

\section{Introduction}

The rhodanese-domain proteins catalyze in vitro the transfer of a sulfur atom from a suitable sulfur donor (thiosulfate for rhodaneses, and 3-mercaptopyruvate for 3-mercaptopyruvate sulfurtransferases) to cyanide, with concomitant formation of thiocyanate (Westley et al., 1983). Their biochemical functions and cellular roles remain unclear despite their presence in all domains of life. Starting from the finding that bovine rhodanese (Nandi and Westley, 1998), Escherichia coli GlpE (Ray et al., 2000), and the 3-mercaptopyruvate sulfurtransferase from Leishmania (Williams et al., 2003) have higher affinity for reduced thioredoxins than for cyanide, the concept that cyanide detoxification was not the only physiological role of the rhodanese-like proteins has emerged. The relevance of specific rhodanese-like proteins in the maintenance of redox homeostasis has been suggested by in vitro studies with rat mercaptopyruvate sulfurtransferase (Nagahara and Katayama, 2005; Nagahara et al., 2007), and proteomic analyses (Krivobok et al., 2003; Santos et al., 2004) indicated possible roles of bacterial rhodanese-domain proteins in physiological processes related to xenobiotic-induced oxidative-stress and detoxification. This experimental evidence suggests that rhodanese domains might function as regulatory devices in specific physiological situations, but the redundancy of these proteins in the same organism make it difficult to define their different cellular roles.

In Azotobacter vinelandii there are 11 open reading frames coding for rhodanese-domain proteins displaying different architectures and active-site motifs (Pfam accession no. PF00581; A. vinelandii DJ subgroup), thus making it conceivable that substrate recognition and biological interactions are driven by the specific active-site structures. The A. vinelandii tandem domain protein RhdA (Colnaghi et al., 1996) contains an active-site motif (HCQTHHR) not currently found in rhodanese-like proteins, that confers structural peculiarity of the environment of its catalytic cysteine residue (Bordo et al., 2000), the only one present in the molecule. RhdA, however, could represent a good model to prove a specific role of a rhodanese-like protein in the aerobe $A$. vinelandii.

Preliminary phenotypic characterizations of an A. vinelandii mutant strain (MV474) in which the rhdA gene was disrupted by deletion demonstrated some metabolic imbalance that pointed to an involvement of RhdA in protecting from inactivation enzymes containing labile Fe-S (Cereda et al., 2007). Accordingly, the defects of the A. vinelandii RhdA null mutant was elicited by using growth conditions that enhance the catalytic deficiency of enzymes with vulnerable Fe-S clusters (Cereda et al., 2009). However, in the presence of gluconate as carbon source, which activates the EntnerDoudoroff pathway (Peekhaus and Conway, 1998) where the first enzyme 6-phosphogluconate dehydratase contains a vulnerable 4Fe-4S cluster (Gardner and Fridovich, 1991; Imlay, 2006), it was found that defensive activities against oxidative damage were needed to rescue the effects of the lack of RhdA in A. vinelandii MV474. Collectively, our previous results suggested that RhdA might trigger protection to oxidative events in $A$. vinelandii, but a link between the absence of RhdA and an altered cellular redox balance in A. vinelandii was still not established.

In the current study, analytical measurements, and transcript profiles provided evidence that during aerobic respiration redox imbalance occurred in $A$. vinelandii cells lacking RhdA. Induction of the $a h p C$ transcript, coding for alkyl hydroperoxide reductase (AhpC), an adaptive response to oxidative stress (Ochsner et al., 2000; Seaver and Imlay, 
2001; Zheng et al., 2001), was observed in MV474. Moreover, an altered activity of the transcription factor CydR, an oxygen sensor with emerging roles in controlling $A$. vinelandii respiration (Setubal et al., 2009), was indicated by the finding that only in MV474 the level of the $c y d A B$ transcript, coding for a cytochrome oxidase negatively controlled by CydR (Wu et al., 1997, 2000), was induced.

Furthermore, in conditions of provoked oxidative stress by addition of the superoxide generator phenazine methosulfate (PMS), a stressor-dependent induction of the rhdA gene provided a link between RhdA and the PMS-resistance of the wild-type strain previously observed (Cereda et al., 2007, 2009). The potentiality of RhdA in buffering the general levels of oxidants in A. vinelandii cells is discussed.

\section{Results}

\section{Effects of the lack of RhdA on cellular redox status of A. vinelandii}

The phenotypic characterization of the A. vinelandii rhdA mutant strain MV474 (Cereda et al., 2007, 2009) clearly showed that inactivation of enzymes containing labile Fe-S clusters (Imlay, 2006) was enhanced in the absence of RhdA. Our goal was, therefore, to explore whether these effects could correlate with an altered redox balance in $A$. vinelandii cells lacking RhdA.

The oxidant-sensitive probe DCFH-DA was at first used to compare the level of reactive oxygen species (ROS) in the wild-type and in the rhdA mutant strain. In both growth conditions (i.e., sucrose, BSN, and gluconate, BGN) levels of endogenously generated ROS (Figure 1A) were higher in mutant than in wild-type cells. The results obtained with MV474 in the presence of gluconate as a carbon source showed a four-fold increase of ROS, whereas a two-fold increase was observed in the presence of sucrose.

Considering that the iron released from damaged $\mathrm{Fe}-\mathrm{S}$ clusters can elicit ROS production via the Fenton reaction (Jang and Imlay, 2007), free iron content was determined by the bathophenanthroline method (Tangeras, 1986). As shown in Figure 1B, in both carbon sources a two-fold increase of iron content, with respect to the level of wild-type extracts, was observed in extracts from A. vinelandii MV474.

Furthermore, the analysis of the levels of glutathione, the most abundant thiol with key roles in protecting cells from oxidative stress (Fahey et al., 1978; Masip et al., 2006), revealed that the percentage of oxidized glutathione was higher in the mutant than in the wild-type $A$. vinelandii (Table 1). Although the relative increases of oxidized glutathione in the mutant were comparable in both growth conditions, the levels of oxidized glutathione were higher in gluconate growths, a condition in which growth defects of MV474 have been observed (Cereda et al., 2009), and ROS production in the absence of RhdA was elicited (Figure 1A; gray bars).
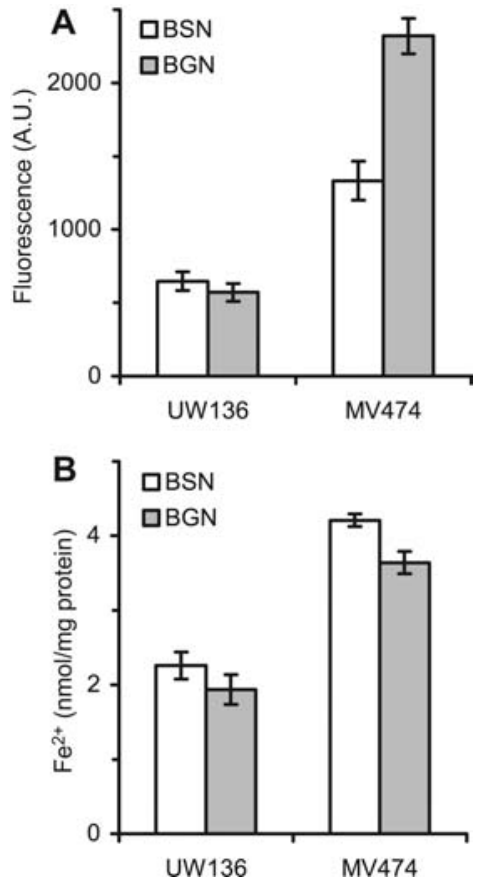

Figure 1 Effects of the lack of RhdA on ROS and free iron levels in A. vinelandii.

Intracellular levels of ROS and free iron were measured in A. vinelandii extracts from wild-type UW136 and rhdA null mutant MV474 strains aerobically grown in BSN or in BGN. (A) ROS generation was measured by the DCFH-DA technique, and the fluorescence of DCF was measured at $519 \mathrm{~nm}$. Values are referred to as arbitrary units (A.U.) of fluorescence after background subtraction. (B) The level of intracellular free iron was measured by the bathophenantroline sulfonate assay, as described under materials and methods. The data shown are the mean \pm SD from three separate growth experiments.

\section{Protective action of RhdA against the superoxide generator PMS}

The analytical picture that has emerged from the above results pointed to a potential link between inactivation of labile $\mathrm{Fe}-\mathrm{S}$ proteins and a redox imbalance generated by deletion of the rhdA gene in A. vinelandii. The first indication that RhdA could protect $A$. vinelandii from oxidative events stemmed from the observation that in the presence of the superoxide generator PMS, A. vinelandii growth was significantly impaired only in the $r h d A$ mutant strain (Cereda et al., 2007, 2009). PMS treatment was, therefore, used to

Table 1 Glutathione disulfide (GSSG) in the A. vinelandii strains UW136 and MV474.

\begin{tabular}{|c|c|c|c|c|}
\hline \multirow[t]{3}{*}{ Strain } & \multicolumn{4}{|c|}{ GSSG (\% of total glutathione) } \\
\hline & \multicolumn{2}{|c|}{ Sucrose ${ }^{a}$} & \multicolumn{2}{|c|}{ Gluconate $^{a}$} \\
\hline & & $(+\mathrm{PMS})$ & & $(+\mathrm{PMS})$ \\
\hline UW136 & $0.73 \pm 0.02$ & $(8.27 \pm 0.08)$ & $2.55 \pm 0.03$ & $(17.81 \pm 0.21)$ \\
\hline MV474 & $1.24 \pm 0.02$ & $(22.38 \pm 0.01)$ & $4.68 \pm 0.05$ & $(82.00 \pm 1.18)$ \\
\hline
\end{tabular}

${ }^{\mathrm{a} C a r b o n}$ source in the growth medium. 
highlight whether the presence of RhdA could buffer the effects of oxidative stress.

As shown in Figure 2, the relative increases of ROS and free iron content, with respect to untreated wild-type strain UW136, were higher in the mutant than in PMS-treated wildtype cells. Collectively, the data shown in Figure 2 proved that in the mutant strain the stress provoked by the presence of PMS during growths was elicited. The relative increase of ROS in MV474 was higher than that of iron, indicating that the growth defect, observed only in PMS-grown MV474, was essentially correlated to an enhanced production of ROS.

The level of oxidized/reduced glutathione in conditions of induced oxidative stress (i.e., in the presence of the stressor PMS) highly increased in both strains (Table 1).

It seems, however, that the lower PMS-sensitivity of the wild-type strain originated by the ability of RhdA to 'buffer' ROS production, and prompted us to investigate whether a stressor-dependent induction of $r h d A$ expression occurred in A. vinelandii. Quantitative RT-PCR was performed, and the mRNA level of $r h d A$ gene was analyzed in cells grown with either sucrose or gluconate as carbon source. The results are shown in Figure 3 taking as a reference the level of rhdA transcript found in untreated sucrose-grown UW136. Under standard cellular respiration the level of $r h d A$ transcript was two-fold higher in gluconate-growths, and in both carbon sources induction of the expression of $r h d A$ by the super-
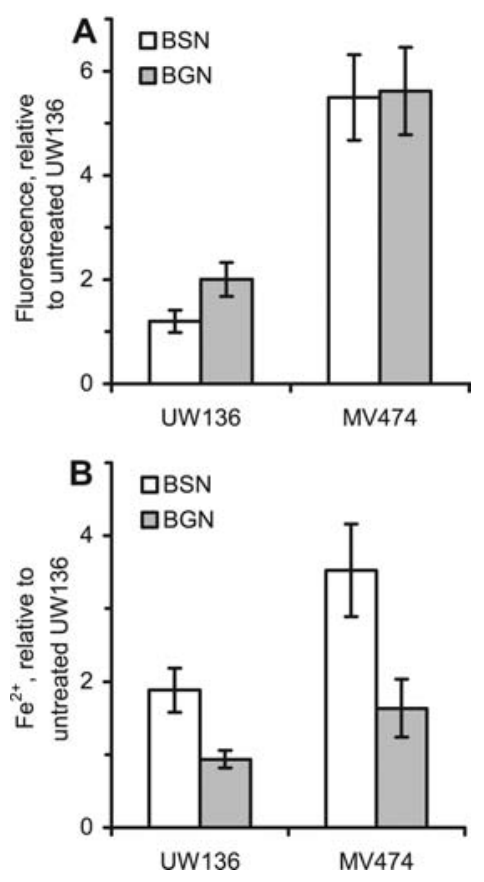

Figure 2 Effects of the lack of RhdA on ROS and free iron levels in A. vinelandii grown in the presence of PMS.

Intracellular levels of ROS (A) and free iron (B) were determined as described in the legend to Figure 1 and under materials and methods. The experimental data are presented as fold increase of ROS and iron levels with respect to those obtained for UW136 grown in BSN in the absence of PMS, and are the mean \pm SD from three separate growth experiments.

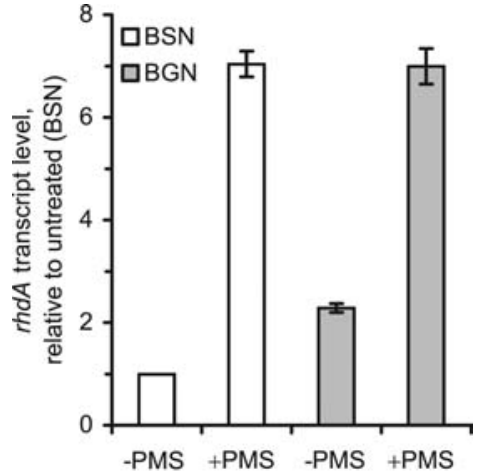

Figure 3 Induction of the $r h d A$ gene in A. vinelandii wild-type UW136 strain.

Quantitative RT-PCR analyses were performed, as described in the materials and methods section, on RNA from A. vinelandii cells grown in BSN and BGN in the absence (-PMS) and in the presence (+PMS) of the stressor PMS. Gene expression was normalized to 16S rRNA expression in each sample. Data are presented as $r h d A$ transcript abundance fold increase with respect to the abundance of the rhdA transcript in cells grown in $\mathrm{BSN}(=1)$, and are the mean \pm SD from three separate growth experiments.

oxide generator PMS was observed. Under conditions of induced oxidative stress, a three-fold and six-fold increase of the expression of $r h d A$ was found in gluconate- and in sucrose-growths, respectively. This finding highlighted the potential role of RhdA in modulating cellular redox events, previously suggested by the observation that defensive activities against oxidative stress were rescued in the absence of RhdA (Cereda et al., 2009). However, in gluconate-growths, although PMS treatment of the $r h d A$ mutant strain primed the overexpression of alkyl hydroperoxide reductase (AhpC), A. vinelandii MV474 did not show resistance to the exposure to PMS comparable with that of the wild-type strain (Cereda et al., 2009).

To better identify a potential link between activation of defensive activities against oxidative damage and RhdA lack in $A$. vinelandii, we performed transcriptomic investigations by using growth conditions (i.e., sucrose as the carbon source) in which, in spite of the proved oxidative imbalance owing to the lack of RhdA (see Figure 1 and Table 1), MV474 grew as well as the wild-type strain UW136 (Cereda et al., 2007). Analysis of the expression of $a h p C$ gene (coding for $\mathrm{AhpC}$ ) revealed that also during cellular respiration the expression of the $a h p C$ transcript in the RhdA mutant was approximately seven-fold higher than in wild-type cells (Figure 4). This result represents a further proof that deletion of the $r h d A$ gene makes $A$. vinelandii more prone to stress, as originally suggested by the observed (Cereda et al., 2007) early accumulation of polyhydroxybutyrate (PHB) in MV474. Accumulation of PHB is considered a 'cell survival mechanism' (Anderson and Dawes, 1990; Ayub et al., 2004), and the same $A$. vinelandii phenotype was observed as a consequence of inactivation of the $c y d R$ gene $(\mathrm{Wu}$ et al., 2001) coding for the transcriptional regulator CydR (GeneID: 7760921; Uniprot acc. no. C1DEL3). Recently, the genome sequence of $A$. vinelandii pointed to a role of CydR 


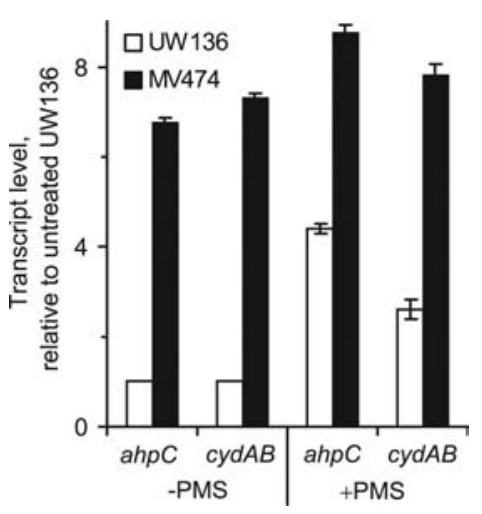

Figure 4 Relative abundance of $a h p C$ and $c y d A B$ transcripts in the A. vinelandii UW136 and MV474 strains.

Quantitative RT-PCR analyses were performed, as described under materials and methods, on RNA from A. vinelandii UW136 and MV474 cells grown in BSN in the absence (-PMS) and in the presence (+PMS) of the stressor PMS. Gene expression was normalized to $16 \mathrm{~S} r R N A$ expression in each sample. Data are presented as ahpC and $c y d A B$ transcript abundance fold increase with respect to the abundance of ahpC and $c y d A B$ transcripts determined in wild-type UW136 strain (PMS-untreated) grown in BSN $(=1)$. The data are the mean $\pm \mathrm{SD}$ from three separate growth experiments.

in regulating various physiological processes associated with respiratory protection (Setubal et al., 2009). CydR can coordinate an exceptionally oxygen-labile Fe-S cluster (Wu et al., 2000) which provides a mechanism for CydR to regulate the expression of respiratory genes (Wu et al., 1997). Considering that cytochrome $b d$ expression is negatively regulated by CydR (Wu et al., 2000), analysis of the $c y d A B$ transcript would provide a link between the integrity of CydR and PHB accumulation in the RhdA null mutant.

As shown in Figure 4, a seven-fold induction of the $c y d A B$ transcript was found in the RhdA mutant strain when compared with the level of $A$. vinelandii wild-type cells. Furthermore, we analyzed whether modulation of $a h p C$ and $c y d A B$ transcripts occurred in $A$. vinelandii wild-type cells under induced oxidative stress.

When growths were performed in the presence of PMS the level of $\operatorname{ahpC}$ transcript in A. vinelandii wild-type strain was four-fold higher than in untreated cells, in line with the induction by PMS of $a h p C$ transcript observed in Escherichia coli (Lee et al., 2004). An increase of the $c y d A B$ transcript was also observed in the PMS-grown wild-type strain, proving a link between PMS-induced damage of the CydR labile Fe-S cluster and expression of $c y d A B$ gene. As for the RhdA mutant, both $a h p C$ and $c y d A B$ transcripts were slightly induced in PMS-growths, when compared with the transcript levels observed in standard growth conditions.

\section{Outlining redox-switch modulation of RhdA}

In line with the level of $r h d A$ transcript (Figure 3), analysis of the expression of RhdA showed that PMS-treatment primed overexpression of this rhodanese-domain protein (Figure 5), thus supporting its potential role in helping $A$. vinelandii to sustain oxidative imbalance.
Scavenging of oxidative species should imply a redoxswitch modulation of RhdA, that could be accomplished via thiol modifications. The reactivity of RhdA $\mathrm{Cys}_{230}-\mathrm{SH}$ (the only one present in the molecule) that can promote ionization, even at neutral $\mathrm{pH}$, to the thiolate anion $\left(\mathrm{Cys}^{-} \mathrm{S}^{-}\right)$was demonstrated in our previous in vitro studies with the purified protein (Cereda et al., 2009). It was found that in oxidative conditions formation of sulfenyl $\left(\mathrm{Cys}_{230}-\mathrm{SOH}\right)$ and sulfinyl ( $\left.\mathrm{Cys}_{230}-\mathrm{SOOH}\right)$ groups on RhdA occurred. The high reactivity and low stability of the potential intermediate sulfenic acid (Kiley and Storz, 2004; Poole et al., 2004), did not allow in vivo detection of reversible modifications of the RhdA thiol.

To study cellular modifications of the RhdA thiol we therefore used the thiosulfate:cyanide sulfurtransferase activity (TST, thiocyanate production) as a probe (Sörbo, 1953), for which the availability of the RhdA $\mathrm{Cys}_{230}$ thiol is mandatory. As expected, considering the level of $r h d A$ transcript and RhdA expression when gluconate was the carbon source (see Figures 3 and 5), TST activity was higher in extracts from gluconate-growths than in those from sucrose-growths (Figure 6A; white bars). To test whether reversible oxidative modifications of the RhdA thiol occurred during A. vinelandii respiration, TST activity was measured in the presence of the thioredoxin/thioredoxin reductase system (Figure 6A; gray bars).

In both growth conditions TST activity of the extracts did not significantly increase upon reducing treatment, indicating
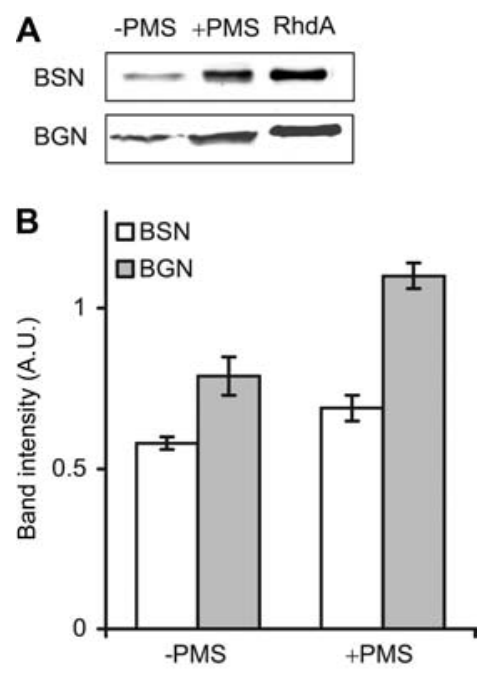

Figure 5 RhdA expression in A. vinelandii UW136 grown in the presence of PMS.

Whole cell lysates of A. vinelandii UW136 grown in BSN or BGN either in the absence or in the presence of PMS were analyzed by SDS-PAGE and Western blotting using an anti-RhdA antibody. (A) Representative Western blot of the lysates from growth in the absence (-PMS) and in the presence (+PMS) of PMS. RhdA, purified recombinant protein (50 ng). (B) Semi-quantification of immunodetected RhdA (referred to as arbitrary units, A.U.) was performed by normalization to both Red Ponceau S intensity of transferred proteins and intensity of purified RhdA. The data presented are the mean \pm SD from three separate growth experiments. 

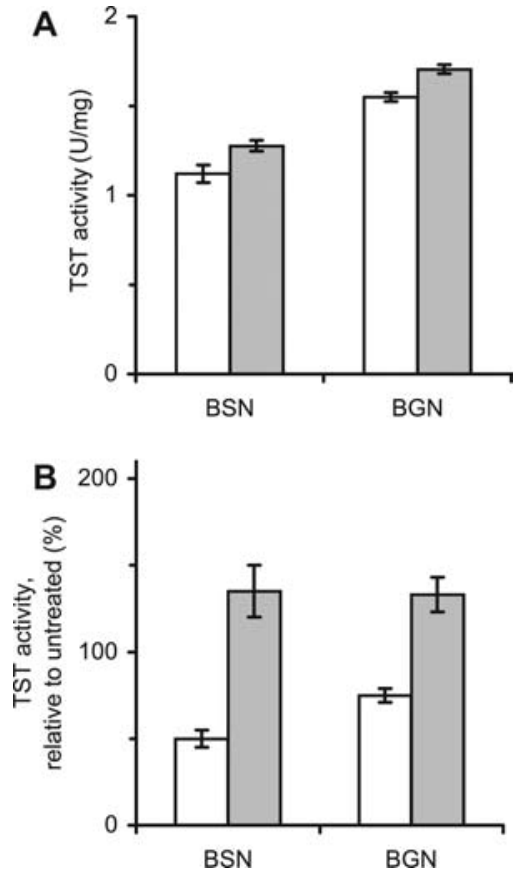

Figure 6 Thiosulfate:cyanide sulfurtransferase (TST) activity in A. vinelandii UW136 cells grown in the absence and in the presence of PMS.

TST activity was measured in cell-free extracts from $A$. vinelandii grown with sucrose $(\mathrm{BSN})$ or gluconate $(\mathrm{BGN})$ as carbon source either in the absence (A) or in the presence (B) of PMS. Modulation of TST activity by pre-incubation of the cell extracts in the presence of thioredoxin/thioredoxin reductase system (gray bars) is reported. TST activity in extracts from PMS-growth is referred to as the percentage of that measured in extracts from cells grown in the absence of PMS. All the data presented are the mean \pm SD from three separate growth experiments.

that during cellular respiration the availability of RhdA thiol was maintained.

When growths were performed in oxidative stress conditions (i.e., in the presence of PMS) the availability of RhdA thiol was affected, and the residual TST activity was approximately $50 \%$ in sucrose- and $75 \%$ in gluconate-growths with respect to untreated cells (Figure 6B; white bars). Treatments in the presence of the thioredoxin/thioredoxin reductase system (Figure 6B; gray bars) of the extracts from PMS-growths allowed activity recovery, thus indicating that the oxidative modifications of RhdA $\mathrm{Cys}_{230}$ can be reversed. In line with the induction of RhdA during growths in the presence of PMS, we observed activity figures higher than $100 \%$ recovery. It is worth noting that the percentage recovery was referred to the TST activity measured in extracts from sucrose- and gluconate-growths in the absence of PMS.

\section{Discussion}

In this study, analytical measurements of parameters generally recognized as indicators of altered cell redox homeosta- sis (D'Autreaux and Toledano, 2007; Jang and Imlay, 2007) revealed that the lack of the rhodanese-domain protein RhdA generates a redox imbalance during cellular respiration of A. vinelandii. The extent to which ROS accumulate is determined by antioxidant systems which enable organisms to maintain proteins and other cellular components in an active state for metabolism (Foyer and Noctor, 2005). Furthermore, the depletion of reduced glutathione (GSH) in MV474, with respect to the level measured in the wild-type strain, proved an altered redox status in A. vinelandii cells lacking RhdA. The GSH/GSSG pool is considered to be a major indicator of the cellular redox status (Foyer and Noctor, 2005), and it is generally recognized that the ratio GSH/GSSG in bacteria is carefully controlled to guarantee a proper cellular redox potential (Fahey et al., 1978), but the mechanisms responsible for GSH homeostasis are still only partially understood.

The present study provides a link between an altered cellular redox balance in A. vinelandii MV474 lacking the rhodanese-like RhdA and overexpression of enzymes induced during oxidative stress (Cunningham et al., 1997; Sandercock and Page, 2008) as previously found for aconitate hydratase 1, and catalase (Cereda et al., 2009). Moreover, the potential of RhdA in controlling the redox homeostasis in A. vinelandii was confirmed by the finding that expression of the rhdA gene was modulated by the growth conditions. Under standard aerobic respiration, in the presence of gluconate as a carbon source $r h d A$ gene expression was higher than in sucrose-growths. Under conditions of induced oxidative stress in both carbon sources $r h d A$ gene was significantly induced. Considering that in these enhanced stress conditions growth rates were impaired only in the A. vinelandii RhdA mutant (Cereda et al., 2007, 2009), it became conceivable that the $r h d A$ gene product has a role in modulating cellular redox events, thus limiting the oxidative imbalance generated by the presence of the superoxide generator PMS. Regulated adaptive responses to oxidative stress have been extensively studied using E. coli as a model organism (Pomposiello and Demple, 2001; Seaver and Imlay, 2001; Zheng et al., 2001), and it is generally recognized that catalase and alkyl hydroperoxide reductase (the product of $\operatorname{ahpC}$ gene) activities are crucial to oxidative stress survival. AhpC is a member of the OxyR regulon, and in E. coli it was found that OxyR can respond to disulfide stress resulting from defects in the systems that maintain an intracellular reducing environment (Aslund et al., 1999). The genome sequence of $A$. vinelandii (Setubal et al., 2009) revealed the presence of an $a h p C$ gene coding for alkyl hydroperoxide reductase (Uniprot acc. no. C1DHW8) showing 74\% sequence identity with E. coli AhpC (Uniprot acc. no. P0AE08). Redundancy of protection mechanisms from oxidative damage seems to be a peculiarity of the aerobe $A$. vinelandii (Setubal et al., 2009), but, to our knowledge, studies devoted to investigate physiological responses to the generation of oxidative species in A. vinelandii are lacking.

In the present study, we performed a transcriptomic investigation, restricted to the analysis of $\operatorname{ahpC}$ and $c y d A B$ transcripts, to compare responses of $A$. vinelandii MV474 and UW136 with the superoxide generator PMS. The finding 
that also in standard aerobic growths the level of both ahpC and $c y d A B$ transcripts was significantly increased in the $r h d A$ mutant (approx. seven-fold as compared with the level of the wild-type strain) deserves some consideration. Expression of respiratory components, including $c y d A B$, is under control of the oxygen-sensitive FNR-like protein CydR for which genomic analyses (Setubal et al., 2009) indicated involvement in the regulation of various processes. Notably, analysis of the $c y d A B$ transcript could provide insights on CydR inactivation, because expression of $c y d A B$ is negatively controlled by this FNR-like Fe-S transcriptional regulator (Wu et al., 1997, 2000). As stated previously, studies on the role of A. vinelandii alkyl hydroperoxide reductase in managing stress tolerance are lacking, but it seems conceivable that its role should be similar to that of other bacterial AhpCs (Aslund et al., 1999; Ochsner et al., 2000; Seaver and Imlay, 2001; Hishinuma et al., 2006). Accordingly, the high level of $a h p C$ transcript in MV474 proved that defensive activities against oxidants are required when RhdA is lacking. The increase of $c y d A B$ transcript in MV474 represents an indication that the oxygen vulnerable Fe-S cluster of CydR (Wu et al., 1997, 2000) was damaged in the absence of RhdA, and it provided a link between the observed PHB accumulation in the RhdA null mutant (Cereda et al., 2007) and CydR inactivation. Under severe conditions of oxidative stress, in line with the ahpC transcript induction by PMS observed in E. coli (Lee et al., 2004), A. vinelandii UW136 also responded to the stressor PMS by increasing $a h p C$ transcript. The level of $c y d A B$ transcript appeared to be modestly modulated by PMS exposure. In the case of the RhdA mutant strain, the effects of PMS in inducing ahpC and $c y d A B$ transcripts, when compared with untreated MV474, were modest, probably because of the high expression of $\operatorname{ahpC}$ and $c y d A B$ transcripts under standard growth conditions.

Collectively, analytical and transcriptomic data proved that deletion of the $r h d A$ gene makes $A$. vinelandii more prone to accumulate ROS, an oxidative stress condition to which A. vinelandii responded by activating defensive mechanisms. The ability of RhdA in maintaining A. vinelandii cellular redox status does imply that RhdA functions as a redox switch, a role that, in principle, might be accomplished by using as a player the highly reactive thiol of its catalytic cysteine residue (Bordo et al., 2000). The key role played by sulfhydryl groups (-SH) in the response to oxidative stress is emerging (Kiley and Storz, 2004; Poole et al., 2004; Jacob et al., 2006). The decreased availability of the RhdA $\mathrm{Cys}_{230^{-}}$ thiol in extracts from PMS-treated A. vinelandii UW136, proved by RhdA activity tests, provided evidence that RhdA $\mathrm{Cys}_{230}$-thiol modifications occurred in vivo. Although additional experimental strategies will be required to determine the pathway of the RhdA thiol ox-red reactions, the finding that the cellular reducing system thioredoxin-mediated was able to reshuffle RhdA thiol is of physiological significance.

In conclusion, the present study proved in vivo the role of a specific rhodanese-domain protein in maintaining cellular redox homeostasis, and could expand the functional classification of the abundant rhodaneses considering the environmental features of their catalytic loop.

\section{Materials and methods}

\section{Bacterial strains and growth conditions}

The A. vinelandii strains used in this study were UW136 and a derivative of UW136 (MV474) in which disruption of the rhdA gene was achieved by the insertion of a KIXX cassette, following deletion of $584 \mathrm{bp}$ as described in Colnaghi et al. (1996). Cells were grown aerobically in Burk's medium for $24 \mathrm{~h}$ at $30^{\circ} \mathrm{C}$, supplemented with $15 \mathrm{~mm}$ ammonium acetate and $1 \%$ sucrose or $0.2 \%$ gluconate. When the optical density reached $\mathrm{OD}_{600}=2.0$, the cells were spun down at $3800 \mathrm{~g}$ for $10 \mathrm{~min}$, and stored at $-80^{\circ} \mathrm{C}$. For oxidative stress induction, cells were grown in the above conditions with the addition of PMS (final concentration $15 \mu \mathrm{M}$ ) at $\mathrm{OD}_{600}=0.800$.

\section{RT-PCR analysis}

Total RNA was isolated using the NucleoSpin ${ }^{\circledR}$ RNA II kit (Macherey-Nagel, Düren, Germany) according to the manufacturer's protocols. Reverse transcription was performed on $1200 \mathrm{ng}$ of total RNA, using RevertAid ${ }^{\mathrm{TM}} \mathrm{H}$ Minus M-MuLV RTase (Fermentas Int. Inc., Burlington, ON, Canada), RiboLock ${ }^{\mathrm{TM}}$ RNAse inhibitor (Fermentas), and reverse primers. Primers were: RhdAWR1FOR (5'ACA ACT GGA AAG CCT GTT CG-3') and RhdAWR1REV (5'-CCT TGG CGA TCA GGT AGG T-3') for the amplification of A. vinelandii rhdA (703771-702956 nucleotide region of GenBank GI: 7759697; amplicon size: 533 bp), 16SFOR (5'-ACC GCA TCC AAA ACT ACT GG-3') and 16SREV (5'-CAC CGG CAG TCT CCT TAG AG-3') for the amplification of A. vinelandii $16 \mathrm{~S}$ rRNAs (177422-178956 nucleotide region of GenBank GI: 7759149; amplicon size: $537 \mathrm{bp}$ ), AhpCFOR (5'-CCT ACC ACA ACG GCA AGT TC- $3^{\prime}$ ) and AhpCREV (5'-AGA TCC AGG GAC GGC TTC$\left.3^{\prime}\right)$ for the amplification of A. vinelandii ahpC (4662884-4663447 nucleotide region of GenBank GI: 7763457; amplicon size: 505 bp), CydBFOR (5'-CTG GGC CTT GCT ACT GGT T-3') and CydBREV (5'-TAG CGC ACG TAG TTG GTC TG-3') for the amplification of A. vinelandii cydAB (1975511-1974372 nucleotide region of GenBank GI: 7760919; amplicon size: 525 bp). Quantitative reverse transcription real-time PCR (qRT-PCR) was carried out using the SYBR ${ }^{\circledR}$ Green Supermix (Bio-Rad, Richmond, CA, USA) in a final volume of $20 \mu \mathrm{l}$. For each reaction the following were added: cDNA (obtained from $2 \mathrm{ng}$ total RNA), $0.25 \mu \mathrm{M}$ of each primer, and $5 \%$ dimethyl sulfoxide (DMSO). Amplification conditions were: $95^{\circ} \mathrm{C}$ for 4 min, followed by 45 cycles consisting of $95^{\circ} \mathrm{C}$ for $40 \mathrm{~s}, 59^{\circ} \mathrm{C}$ for $40 \mathrm{~s}, 72^{\circ} \mathrm{C}$ for $40 \mathrm{~s}$. Each qRT-PCR was performed in triplicate. Negative controls were performed with $2 \mathrm{ng}$ of non-reverse transcribed RNA as a template, and in the absence of a template. In positive controls, A. vinelandii genomic DNA (190 ng, extracted from A. vinelandii cell cultures using a proteinase $\mathrm{K}$-based minipreparation) was used as a template. Data were elaborated according to Livak and Schmittgen (2001) using $16 S$ rRNA as a reference.

\section{Analytical procedures}

Cell-free extracts of $A$. vinelandii were prepared by sonication (five 30-s pulses with intermittant 1-min cooling periods in Soniprep 150 (MSE, London, UK) in $10 \mathrm{~mm}$ Tris- $\mathrm{HCl}, 100 \mathrm{~mm} \mathrm{NaCl}$ (pH 8), and cell debris was removed by centrifugation (30 min at $10000 \mathrm{~g}$ ). The protein concentration was determined by the Bradford assay (Bradford, 1976) using bovine serum albumin as a standard.

Thiosulfate:cyanide sulfurtransferase (TST) activity was tested by the discontinuous method described by Sörbo (1953). Where it is stated, before enzyme assay, cell-free extract samples (100-200 $\mu \mathrm{g}$ 
of proteins) were incubated in $20 \mathrm{~mm}$ phosphate buffer ( $\mathrm{pH} 7.4)$, $50 \mu \mathrm{M}$ E. coli reduced thioredoxin (Trx; Sigma-Aldrich S.r.l., Milan, Italy), $0.2 \mu \mathrm{M}$ rat liver thioredoxin reductase (Sigma-Aldrich) and $100 \mu \mathrm{M}$ NADPH at room temperature for $30 \mathrm{~min}$. One unit of TST activity (U) is defined as the amount of enzyme that produces $1 \mu \mathrm{mol}$ thiocyanate in $1 \mathrm{~min}$ at $37^{\circ} \mathrm{C}$.

Chelatable iron (free iron) was determined according to Tangeras (1986). Cell-free extracts (approx. $400 \mu \mathrm{g}$ total protein) were prepared in $15 \mathrm{mM} \mathrm{KH}_{2} \mathrm{PO}_{4}, 150 \mathrm{~mm} \mathrm{NaCl}$ (pH 7.4) (PBS) containing $15 \mathrm{~mm}$ DTT, and incubated in the presence of $0.2 \mathrm{~mm}$ bathophenantroline sulfonate (BPS) and $10 \mathrm{~mm}$ sodium dithionite at room temperature $\left(24^{\circ} \mathrm{C}\right)$ for $15 \mathrm{~min}$. Absorbance was recorded at $535 \mathrm{~nm}$ $\left(\varepsilon_{535}=25100 \mathrm{M}^{-1} \mathrm{~cm}^{-1}\right)$, and data were corrected for protein concentration.

Denaturing gel electrophoresis (SDS-PAGE) was done according to Laemmli (1970) by using the whole cell lysates obtained by heat denaturation of cellular pellets in Laemmli buffer containing $0.35 \mathrm{M}$ 2-mercaptoethanol. Western blot analyses were carried out by a standard protocol using anti-RhdA antiserum (Cereda et al., 2007). Coomassie Blue-stained gels, Red Ponceau S-stained blotted membranes, and immunolabeled membranes were digitized with an Expression 1680 Pro scanner (Epson Italia S.p.A., Milan, Italy), and densitometric analyses were performed with Image Master ${ }^{\mathrm{TM}}-1 \mathrm{D}$ Elite (Amersham Pharmacia Biotech, Little Chalfort, UK).

Measurement of the levels of glutathione was performed by a monobromobimane HPLC method (Riemenschneider et al., 2005) using $50 \mathrm{mg}$ (fresh weight; f.w.) cell samples. Oxidized glutathione was measured using $120 \mathrm{mg}$ (f.w.) cell samples in a Beckman P/ ACE MDQ capillary electrophoresis system (Palo Alto, CA, USA) equipped with a UV absorbance detector set at $200 \mathrm{~nm}$ according to Muscari et al. (1998). The fused-silica capillary $(65 \mathrm{~cm} \times 75 \mu \mathrm{m}$ internal diameter) was maintained at $28^{\circ} \mathrm{C}$. Samples were injected by pressure $(2.07 \mathrm{kPa})$ for $10 \mathrm{~s}$ and separated at $30 \mathrm{kV}$. The background electrolyte contained $100 \mathrm{~mm}$ boric acid and $25 \mathrm{~mm}$ Tris ( $\mathrm{pH}$ 8.2). Data were collected and processed with 32 Karat $^{\mathrm{TM}}$ Beckman software.

\section{Measurement of ROS levels}

The intracellular ROS levels in UW136 and MV474 cultures were determined using $2^{\prime}, 7^{\prime}$-dichlorodihydrofluorescein diacetate (DCFHDA, Molecular Probes, OR, USA) (Echave et al., 2003). DCFH-DA is cleaved by cellular esterases to $2^{\prime}, 7^{\prime}$-dichlorodihydrofluorescein $(\mathrm{DCFH})$, and oxidation of DCFH by intracellular ROS gives raise to the fluorescent product $2^{\prime}, 7^{\prime}$-dichlorofluorescein (DCF). Cells were collected by mild centrifugation $(3800 \mathrm{~g}, 10 \mathrm{~min})$, washed using PBS, and, before the addition of DCFH-DA (dissolved in DMSO), cell density was adjusted to $\mathrm{A}_{600}=0.4$. After 30 min incubation with $10 \mu \mathrm{M}$ DCFH-DA at $30^{\circ} \mathrm{C}$, cells were washed in PBS, resuspended in $3 \mathrm{ml} \mathrm{PBS}$, and sonicated (five 30 -s pulses with intermittant 1-min cooling periods in Soniprep 150, UK). Fluorescence of DCF was immediately detected using a Perkin Elmer LS-50 spectrofluorimeter $\left(\lambda_{\mathrm{exc}}=490 \mathrm{~nm} ; \lambda_{\mathrm{em}}=519 \mathrm{~nm}\right.$; excitation and emission slits $=3 \mathrm{~nm}$ ). In the negative control, DCFH-DA was omitted, and the final concentration of DMSO (the solvent in the probe stock solution) was maintained equal to the treated samples. Fluorescence values were normalized by protein concentration.

\section{Acknowledgments}

Mobility of researchers between Italy and Germany was sponsored by Vigoni project n. 0815171 (2009-2010; Ateneo Italo-Tedesco,
Deutscher Akademischer Austauschdienst) to F.F. and J.P. We thank Dr. Aristodemo Carpen for skilful technical assistance.

\section{References}

Anderson, A.J. and Dawes, E.A. (1990). Occurrence, metabolism, metabolic role, and industrial uses of bacterial polyhydroxyalkanoates. Microbiol. Rev. 54, 450-472.

Aslund, F., Zheng, M., Beckwith, J., and Storz, G. (1999). Regulation of the OxyR transcription factor by hydrogen peroxide and the cellular thiol-disulfide status. Proc. Natl. Acad. Sci. USA 96, 6161-6165.

Ayub, N.D., Pettinari, M.J., Ruiz, J.A., and Lopez, N.I. (2004). A polyhydroxybutyrate-producing Pseudomonas sp. isolated from Antarctic environments with high stress resistance. Curr. Microbiol. 49, 170-174.

Bordo, D., Deriu, D., Colnaghi, R., Carpen, A., Pagani, S., and Bolognesi, M. (2000). The crystal structure of a sulfurtransferase from Azotobacter vinelandii highlights the evolutionary relationship between the rhodanese and phosphatase enzyme families. J. Mol. Biol. 298, 691-704.

Bradford, M.M. (1976). A rapid and sensitive method for the quantitation of microgram quantities of protein utilizing the principle of protein-dye binding. Anal. Biochem. 72, 248-254.

Cereda, A., Carpen, A., Picariello, G., Iriti, M., Faoro, F., Ferranti, P., and Pagani, S. (2007). Effects of the deficiency of the rhodanese-like protein RhdA in Azotobacter vinelandii. FEBS Lett. 581, 1625-1630.

Cereda, A., Carpen, A., Picariello, G., Tedeschi, G., and Pagani, S. (2009). The lack of rhodanese RhdA affects the sensitivity of Azotobacter vinelandii to oxidative events. Biochem. J. 418, 135-143.

Colnaghi, R., Pagani, S., Kennedy, C., and Drummond, M. (1996). Cloning, sequence analysis and overexpression of the rhodanese gene of Azotobacter vinelandii. Eur. J. Biochem. 236, 240-248.

Cunningham, L., Gruer, M.J., and Guest, J.R. (1997). Transcriptional regulation of the aconitase genes (acnA and $\mathrm{acnB}$ ) of Escherichia coli. Microbiology 143, 3795-3805.

D'Autreaux, B. and Toledano, M.B. (2007). ROS as signalling molecules: mechanisms that generate specificity in ROS homeostasis. Nat. Rev. Mol. Cell Biol. 8, 813-824.

Echave, P., Tamarit, J., Cabiscol, E., and Ros, J. (2003). Novel antioxidant role of alcohol dehydrogenase E from Escherichia coli. J. Biol. Chem. 278, 30193-30198.

Fahey, R.C., Brown, W.C., Adams, W.B., and Worsham, M.B. (1978). Occurrence of glutathione in bacteria. J. Bacteriol. 133, $1126-1129$.

Foyer, C.H. and Noctor, G. (2005). Redox homeostasis and antioxidant signaling: a metabolic interface between stress perception and physiological responses. Plant Cell 17, 1866-1875.

Gardner, P.R. and Fridovich, I. (1991). Superoxide sensitivity of the Escherichia coli 6-phosphogluconate dehydratase. J. Biol. Chem. 266, 1478-1483.

Hishinuma, S., Yuki, M., Fujimura, M., and Fukumori, F. (2006). OxyR regulated the expression of two major catalases, KatA and KatB, along with peroxiredoxin, AhpC in Pseudomonas putida. Environ. Microbiol. 8, 2115-2124.

Imlay, J.A. (2006). Iron-sulphur clusters and the problem with oxygen. Mol. Microbiol. 59, 1073-1082.

Jacob, C., Knight, I., and Winyard, P.G. (2006). Aspects of the 
biological redox chemistry of cysteine: from simple redox responses to sophisticated signalling pathways. Biol. Chem. 387, $1385-1397$.

Jang, S. and Imlay, J.A. (2007). Micromolar intracellular hydrogen peroxide disrupts metabolism by damaging iron-sulfur enzymes. J. Biol. Chem. 282, 929-937.

Kiley, P.J. and Storz, G. (2004). Exploiting thiol modifications. PLoS Biol. 2, e400.

Krivobok, S., Kuony, S., Meyer, C., Louwagie, M., Willison, J.C., and Jouanneau, Y. (2003). Identification of pyrene-induced proteins in Mycobacterium sp. strain 6PY1: evidence for two ringhydroxylating dioxygenases. J. Bacteriol. 185, 3828-3841.

Laemmli, U.K. (1970). Cleavage of structural proteins during the assembly of the head of bacteriophage T4. Nature 227, 680-685.

Lee, J.H., Yeo, W.S., and Roe, J.H. (2004). Induction of the sufA operon encoding Fe-S assembly proteins by superoxide generators and hydrogen peroxide: involvement of OxyR, IHF and an unidentified oxidant-responsive factor. Mol. Microbiol. 51, $1745-1755$.

Livak, K.J. and Schmittgen, T.D. (2001). Analysis of relative gene expression data using real-time quantitative PCR and the $2^{(-\Delta \Delta C T)}$ method. Methods 25, 402-408.

Masip, L., Veeravalli, K., and Georgiou, G. (2006). The many faces of glutathione in bacteria. Antioxid. Redox Signal. 8, 753-762.

Muscari, C., Pappagallo, M., Ferrari, D., Giordano, E., Capanni, C., Caldarera, C.M., and Guarnieri, C. (1998). Simultaneous detection of reduced and oxidized glutathione in tissues and mitochondria by capillary electrophoresis. J. Chromatogr. B Biomed. Sci. Appl. 707, 301-307.

Nagahara, N. and Katayama, A. (2005). Post-translational regulation of mercaptopyruvate sulfurtransferase via a low redox potential cysteine-sulfenate in the maintenance of redox homeostasis. J. Biol. Chem. 280, 34569-34576.

Nagahara, N., Yoshii, T., Abe, Y., and Matsumura, T. (2007). Thioredoxin-dependent enzymatic activation of mercaptopyruvate sulfurtransferase. An intersubunit disulfide bond serves as a redox switch for activation. J. Biol. Chem. 282, 1561-1569.

Nandi, D.L. and Westley, J. (1998). Reduced thioredoxin as a sulfuracceptor substrate for rhodanese. Int. J. Biochem. Cell Biol. 30, 973-977.

Ochsner, U.A., Vasil, M.L., Alsabbagh, E., Parvatiyar, K., and Hassett, D.J. (2000). Role of the Pseudomonas aeruginosa oxyRrecG operon in oxidative stress defense and DNA repair: OxyR-dependent regulation of katB-ankB, ahpB, and ahpCahpF. J. Bacteriol. 182, 4533-4544.

Peekhaus, N. and Conway, T. (1998). What's for dinner? EntnerDoudoroff metabolism in Escherichia coli. J. Bacteriol. 180, 3495-3502.

Pomposiello, P.J. and Demple, B. (2001). Redox-operated genetic switches: the SoxR and OxyR transcription factors. Trends Biotechnol. 19, 109-114.

Poole, L.B., Karplus, P.A., and Claiborne, A. (2004). Protein sulfenic acids in redox signaling. Annu. Rev. Pharmacol. Toxicol. 44, 325-347.
Ray, W.K., Zeng, G., Potters, M.B., Mansuri, A.M., and Larson, T.J. (2000). Characterization of a 12-kilodalton rhodanese encoded by glpE of Escherichia coli and its interaction with thioredoxin. J. Bacteriol. 182, 2277-2284.

Riemenschneider, A., Nikiforova, V., Hoefgen, R., De Kok, L.J., and Papenbrock, J. (2005). Impact of elevated $\mathrm{H}_{2} \mathrm{~S}$ on metabolite levels, activity of enzymes and expression of genes involved in cysteine metabolism. Plant Physiol. Biochem. 43, 473-483.

Sandercock, J.R. and Page, W.J. (2008). RpoS expression and the general stress response in Azotobacter vinelandii during carbon and nitrogen diauxic shifts. J. Bacteriol. 190, 946-953.

Santos, P.M., Benndorf, D., and Sa-Correia, I. (2004). Insights into Pseudomonas putida KT2440 response to phenol-induced stress by quantitative proteomics. Proteomics 4, 2640-2652.

Seaver, L.C. and Imlay, J.A. (2001). Alkyl hydroperoxide reductase is the primary scavenger of endogenous hydrogen peroxide in Escherichia coli. J. Bacteriol. 183, 7173-7181.

Setubal, J.C., Dos Santos, P., Goldman, B.S., Ertesvag, H., Espin, G., Rubio, L.M., Valla, S., Almeida, N.F., Balasubramanian, D., Cromes, L., et al. (2009). Genome sequence of Azotobacter vinelandii, an obligate aerobe specialized to support diverse anaerobic metabolic processes. J. Bacteriol. 191, 4534-4545.

Sörbo, B.H. (1953). Crystalline rhodanese. Purification and physicochemical examination. Acta Chem. Scand. 7, 1129-1136.

Tangeras, A. (1986). Lysosomes, but not mitochondria, accumulate iron and porphyrins in porphyria induced by hexachlorobenzene. Biochem. J. 235, 671-675.

Westley, J., Adler, H., Westley, L., and Nishida, C. (1983). The sulfurtransferases. Fundam. Appl. Toxicol. 3, 377-382.

Williams, R.A., Kelly, S.M., Mottram, J.C., and Coombs, G.H. (2003). 3-Mercaptopyruvate sulfurtransferase of Leishmania contains an unusual C-terminal extension and is involved in thioredoxin and antioxidant metabolism. J. Biol. Chem. 278, 14801486.

Wu, G., Hill, S., Kelly, M.J., Sawers, G., and Poole, R.K. (1997). The $c y d R$ gene product, required for regulation of cytochrome $b d$ expression in the obligate aerobe Azotobacter vinelandii, is an Fnr-like protein. Microbiology 143, 2197-2207.

Wu, G., Cruz-Ramos, H., Hill, S., Green, J., Sawers, G., and Poole, R.K. (2000). Regulation of cytochrome $b d$ expression in the obligate aerobe Azotobacter vinelandii by CydR (Fnr). Sensitivity to oxygen, reactive oxygen species, and nitric oxide. J. Biol. Chem. 275, 4679-4686.

Wu, G., Moir, A.J., Sawers, G., Hill, S., and Poole, R.K. (2001). Biosynthesis of poly- $\beta$-hydroxybutyrate (PHB) is controlled by CydR (Fnr) in the obligate aerobe Azotobacter vinelandii. FEMS Microbiol. Lett. 194, 215-220.

Zheng, M., Wang, X., Templeton, L.J., Smulski, D.R., LaRossa, R.A., and Storz, G. (2001). DNA microarray-mediated transcriptional profiling of the Escherichia coli response to hydrogen peroxide. J. Bacteriol. 183, 4562-4570.

Received January 29, 2010; accepted March 6, 2010 